Artículo original

\title{
Caracterización de los pacientes con bacteriemia por Staphylococcus aureus resistente a la meticilina en un hospital militar de alta complejidad
}

\author{
Natalie Náder $1,2,3$, Ramón Iván Medina ${ }^{1,2,3}$, Luz Ángela Pescador ${ }^{3}$, Barbarita María \\ Mantilla $^{1,2,3}$, Juan Sebastián Bravo ${ }^{1,2,3}$, Carlos Hernando Gómez ${ }^{3,4}$ \\ ${ }^{1}$ Servicio de Medicina Interna, Hospital Militar Central, Bogotá, D.C., Colombia \\ ${ }^{2}$ Facultad de Medicina, Universidad Militar Nueva Granada, Bogotá, D.C., Colombia \\ ${ }^{3}$ Grupo de Investigación en Enfermedades Infecciosas, Hospital Militar Central, Bogotá, D.C., Colombia \\ ${ }^{4}$ Servicio de Infectología, Hospital Militar Central, Bogotá, D.C., Colombia
}

Introducción. En las Fuerzas Militares de Colombia, cerca de 500.000 de sus miembros asisten a consulta en los establecimientos sanitarios militares. En esta población, Staphylococcus aureus resistente a la meticilina (SAMR) se ha convertido en un agente patógeno de gran incidencia.

Objetivo. Caracterizar los pacientes con diagnóstico de bacteriemia por SAMR en el Hospital Militar Central entre el 2012 y el 2015.

Materiales y métodos. Se llevó a cabo un estudio observacional descriptivo de revisión retrospectiva de historias clínicas de pacientes mayores de 18 años, hospitalizados y con hemocultivos positivos para $S$. aureus resistente a la meticilina. Para la identificación de los pacientes se empleó el sistema Whonet, version 5.6.

Resultados. De los 177 cultivos positivos para $S$. aureus, el $24,8 \%(n=44)$ correspondió a SAMR, con mayor prevalencia en pacientes militares activos( $n=20 m 45,4 \%)$. Se observó una frecuencia similar para la bacteriemia por SAMR adquirida en la comunidad y la adquirida en el hospital, siendo más frecuente $(n=37,84 \%)$ el fenotipo de la comunidad en ambos grupos. El principal foco infeccioso fueron los tejidos blandos, seguidos por el tejido pulmonar. Se presentaron mayores tasas de complicaciones $(61 \%, n=13)$ en la bacteriemia adquirida en el hospital; 34,9\% $(n=15)$, de los pacientes tuvieron una estancia hospitalaria prolongada atribuible a las complicaciones desencadenadas por la bacteriemia.

Conclusiones. La población más afectada por SAMR fueron los pacientes militares activos $(n=20,45,4 \%)$, con una frecuencia similar de la bacteriemia adquirida en la comunidad $(n=18,43,2 \%)$ y la adquirida en el hospital $(n=25,56,8 \%)$, y el principal foco infeccioso fueron los tejidos blandos. Dados estos resultados, es necesario adelantar estudios para establecer la prevalencia de infecciones por SAMR en la piel.

Recibido: 06/09/17

Aceptado: $29 / 08 / 18$

Publicado: $03 / 09 / 18$

Citación:

Náder N, Medina RI, Pescador LA, Mantilla BM, Bravo JS, Gómez CH. Caracterización de pacientes con bacteriemia por Staphylococcus aureus resistente a la meticilina en un hospital militar de alta complejidad. Biomédica. 2019;39(Supl.1):86-95 https://doi.org/10.7705/biomedica.v39i2.4072

Correspondencia:

Juan Sebastián Bravo, Servicio de Medicina Interna, Hospital Militar Central, Transversal $3 \mathrm{~N}^{\circ}$ 49-00,

Bogotá, D.C., Colombia

Teléfono: (300) 4759915

jbravoojeda@gmail.com

Contribución de los autores:

Todos los autores participaron en la adquisición, análisis e interpretación de datos e hicieron contribuciones sustanciales a la concepción y el diseño del manuscrito.

\section{Financiación:}

Los autores declaran no haber recibido ningún tipo de financiación para la realización del presente manuscrito.

\section{Conflicto de intereses:}

Los autores declaran no tener conflictos de intereses.
Palabras clave: Staphylococcus aureus resistente a meticilina; bacteriemia; infecciones de los tejidos blandos; resistencia a medicamentos; personal militar

Characterization of patients with bacteremia by methicillin-resistant Staphylococcus aureus in a high-complexity military hospital

Introduction: In Colombia, there are about 500,000 members in the national armed forces who consult military health institutions. In this population, methicillin-resistant Staphylococcus aureus (MRSA) has become a high-incidence pathogen.

Objective: The aim of this study was to characterize patients with MRSA bacteremia in the Hospital Militar Central between 2012 and 2015.

Materials and methods: This was an observational descriptive study with a retrospective review of clinical histories of hospitalized patients older than 18 years of age with positive blood cultures for methicillin-resistant $S$. aureus. The identification of the patients was made using the Whonet system, version 5.6.

Results: From cultures positive for $S$. aureus, $24.8 \%$ were methicillin-resistant strains, with a higher prevalence in active military personnel. A similar frequency was observed for community-acquired MRSA bacteremias and those acquired at the hospital, with the community phenotype being the most frequent in both groups. The main infectious focus related to the development of bacteremia was soft tissue, followed by pulmonary tissue. There were higher complication rates in nosocomial bacteremias; $34.9 \%$ of the patients had prolonged stays attributable to complications triggered by the bacteremia.

Conclusions: Active military personnel was the most affected population by MRSA, with a similar frequency in community-acquired and nosocomial bacteremias. The main infectious focus was soft tissue. Taking into account these data, studies that establish the prevalence of skin infections by MRSA should be carried out. 
Keywords: Methicillin-resistant Staphylococcus aureus; bacteremia; soft tissue infections; drug resistance; military personnel.

Staphylococcus aureus es conocido como responsable de una gran variedad de enfermedades con un espectro infeccioso amplio que puede ir desde afecciones cutáneas leves hasta infecciones que entrañan una amenaza para la vida, como la neumonía, la endocarditis infecciosa, el síndrome de choque tóxico, la bacteriemia y la sepsis, entre otras (1).

En Colombia, ha habido cambios en la epidemiología de $S$. aureus resistente a la meticilina (SAMR). Según informes del Grupo para el Control de la Resistencia Bacteriana de Bogotá (GREBO), entre el 2008 y el 2016, se apreció una tendencia sostenida a la disminución, y hoy la tasa de resistencia a oxacilina se acerca al $21,4 \%$ en las unidades de cuidados intensivos y a $34,5 \%$ en adultos hospitalizados. Sin embargo, los perfiles genotípicos tienen una gran variabilidad (2). Jimenez, et al., demostraron la presencia de SAMR adquirido en la comunidad (Community Acquired Methicilin Resistant Staphylococcus aureus, CA-MRSA) en un estudio de corte transversal en tres instituciones de alta complejidad de Medellín, con similar frecuencia en aislamientos provenientes de la comunidad y en los de origen hospitalario (46,8 y 40,9\%, respectivamente), lo que significa una condición endémica de este patrón de resistencia fenotípico a nivel local, donde prevalece el clon relacionado USA-300 (3).

El tratamiento de las infecciones por SAMR representa un reto importante, ya que el microorganismo se ha reportado como causa común de bacteriemia adquirida en la comunidad y también asociada con la atención en la salud $(4,5)$, lo que afecta los sistemas de salud e incrementa la incidencia y las complicaciones que, a pesar del uso de nuevos antibióticos, provocan una mortalidad cercana al $30 \%$ (6).

Asimismo, se han descrito factores de riesgo como el aumento de la resistencia a los antibióticos, la frecuencia de cirugías invasivas, el uso de dispositivos endovasculares, la inmunosupresión (pacientes con HIV o con trasplantes, o en tratamiento contra el cáncer), la edad mayor de 65 años y el retraso en el inicio del tratamiento antibiótico apropiado (6-8). Esta alta tasa de mortalidad genera un exceso en los costos y el uso de los recursos de los sistemas de atención en salud, debido a la gran probabilidad de complicaciones que amenazan la vida (9-11).

En Colombia, hay cerca de 500.000 miembros de las Fuerzas Militares, la mayoría de ellos mujeres y hombres jóvenes, que son atendidos en los establecimientos sanitarios militares. En este grupo de pacientes, SAMR se ha convertido en un agente patógeno de gran incidencia.

En este contexto, el objetivo de este estudio fue caracterizar clínica y demográficamente a la población mayor de 18 años atendida en el Hospital Militar Central de Bogotá con diagnóstico de bacteriemia por SAMR en el periodo transcurrido entre 2012 y 2015.

\section{Materiales y métodos}

Se llevó a cabo un estudio observacional descriptivo en el que se revisaron retrospectivamente las historias clínicas de los pacientes mayores de 18 años con hemocultivos positivos para $S$. aureus. 


\section{Lugar}

Los pacientes estuvieron hospitalizados en un hospital militar de alta complejidad de Bogotá, con una capacidad de 520 camas, para la población militar y sus familiares. En el hospital se atienden, aproximadamente, 37.000 consultas externas, 8.000 pacientes de urgencias y se hacen 1.500 cirugías mensualmente.

\section{Selección de pacientes}

Mediante la consulta de la base de datos de microbiología con el programa Whonet, versión 5.6, y utilizando las normas de The Clinical and Laboratory Standards Institute (CLSI) de 2016, se revisaron las historias clínicas y se seleccionaron aquellos 177 pacientes con hemocultivos positivos para SAMR en el periodo comprendido entre 2012 y 2015.

Se excluyeron aquellos pacientes con hospitalizaciones durante la semana anterior y hasta 48 horas antes del inicio de los síntomas en instituciones diferentes al Hospital Militar. Una vez obtenidos los datos, se analizaron los resultados y se describieron las características demográficas y clínicas de la población.

\section{Definiciones}

Bacteriemia adquirida en la comunidad: pacientes con un primer hemocultivo positivo desde su ingreso y hasta 48 horas después, que no cumplieran con alguno de los siguientes criterios: residencia en un hogar geriátrico o centro de cuidados a largo plazo, necesidad de hemodiálisis crónica, tratamiento intravenoso domiciliario, presencia de dispositivos intravasculares a largo plazo y procedimiento invasivo con necesidad de hospitalización en los 90 días anteriores.

Bacteriemia adquirida en el hospital: aquellos pacientes con un primer hemocultivo positivo después de dos días de su hospitalización.

Presencia de catéter central: uso del dispositivo desde 48 horas antes del inicio de los síntomas.

Exposición a antibióticos: uso de antibióticos en los 90 días previos al diagnóstico de bacteriemia.

Fenotipo comunitario (CA-MRSA): según Popovich, et al. (12), se trata de un aislamiento de $S$. aureus resistente a la meticilina, condición establecida mediante una regla de predicción que se correlaciona con la sensibilidad a la clindamicina, $95 \%$ (especificidad=80 \%; LR (likelihood ratio) $=4,86 ; I^{2}{ }_{95 \%} 3,29$ $-6,47$ ) y sensibilidad a la ciprofloxacina, $73 \%$ (especificidad=86 \%; LR=5,34; $\mathrm{IC}_{95 \%}$ 2,91-10,58). Si, además, se presenta en un aislamiento en las primeras 72 horas de hospitalización, no se conoce el índice de probabilidad; en su lugar, se estima el likelihood ratio a $(\mathrm{LR}=10,08)$.

\section{Análisis}

Se calcularon los porcentajes de las variables cualitativas y las medidas de tendencia central, con sus respectivas medidas de dispersión para las variables cuantitativas. Los resultados se obtuvieron utilizando el paquete estadístico Stata $12^{\mathrm{TM}}$. 


\section{Consideraciones éticas}

Por la naturaleza retrospectiva del estudio, así como por no haberse hecho ninguna intervención en los pacientes, este se considera un estudio sin riesgo. Se garantizó la confidencialidad de los datos clínicos de los pacientes en cumplimiento del Artículo 11 de la Resolución 8430 de 1993 expedida por el Ministerio de Salud. El estudio fue aprobado por el Comité de Ética del Hospital Militar.

\section{Resultados}

Se revisaron las historias clínicas de 177 pacientes con hemocultivos positivos para $S$. aureus, de los cuales 44 presentaron bacteriemia por SAMR. Se asignan los porcentajes para facilitar la evaluación e interpretación de los resultados. La edad promedio fue de $49 \pm 23$ años, la mayoría ( $n=30$, $68,1 \%)$ eran hombres. En cuanto a la ocupación, 20 (45,4\%) eran militares activos, $9(20,4 \%)$ eran retirados y $15(34,1 \%)$ eran civiles.

Con respecto a la caracterización de SAMR, 56,8 \% $(n=25)$ de los pacientes presentó bacteriemias adquiridas en el ámbito hospitalario, el restante 43,1 $\%(n=19)$ las habían contraído en la comunidad. Según el perfil de resistencia de SAMR aislados en 44 de los hemocultivos, 37 (84,1\%), correspondían al fenotipo de la comunidad (CA-MRSA) y $7(15,9 \%)$, al fenotipo hospitalario (Hospital Acquired Methicilin Resistant Staphylococcus aureus, HA-MRSA). El fenotipo CA-MRSA se encontró en 78,9\% $(n=15)$ de las bacteriemias originadas en la comunidad y en $88 \%(n=22)$ de las hospitalarias, en tanto que el fenotipo HA-MRSA se encontró en $21 \%(n=4)$ de las bacteriemias originadas en la comunidad y en $12 \%(n=3)$ de las de origen hospitalario.

Entre las variables asociadas con la bacteriemia, se incluyeron la duración de la hospitalización desde el momento de la infección, definida como el número de días transcurrido hasta la toma de los hemocultivos. Se halló una mediana de 9 días, con un mínimo de 0 y un máximo de 80 días. En cuanto al origen de la bacteriemia por SAMR, se encontró que $18(40,9 \%)$ se relacionaba con la sepsis de tejidos blandos, seguida por la neumonía, con $25 \%(n=11)$ de los casos.

En la figura 1 se presenta la distribución según el foco de infección y su lugar de origen, comunidad $u$ hospital. Entre los militares activos, el foco de infección fue más frecuente en los tejidos blandos, seguidos del tejido pulmonar (neumonía) (figura 2).

De los pacientes diagnóstico de bacteriemia por SAMR, el 27,2 \% ( $n=12)$ tuvo exposición previa a antibióticos, principalmente a carbapenémicos $(n=7$, $9,1 \%$ ), seguidos de penicilina ( $n=4,6,8 \%$ ), cefalosporinas, vancomicina y quinolonas, con el 2,22 \% ( $n=2)$ cada uno. Se encontró que 25 pacientes $(56,8 \%)$ presentaron algún tipo de comorbilidad. Se detectó inmunosupresión en $15,6 \%(n=4): 9,0 \%(n=2)$ por quimioterapia y, por uso de corticoides, HIV o neutropenia, cada uno con el $2,2 \%(n=1)$, diabetes mellitus en $20,4 \%$ $(n=5)$, neoplasia hematológica en 6,8 \% $(n=3)$, tumor sólido en $13,6 \%(n=3)$ e insuficiencia renal (sin terapia de reemplazo) en 18,2\% ( $n=4)$. El 47,7\% $(n=21)$ de los pacientes presentaron complicaciones y, de este porcentaje, en $61 \%$ $(n=13)$ las bacteriemias se habían adquirido en el ámbito hospitalario.

En $38 \%(n=8)$ de los casos de bacteriemia adquirida en la comunidad se presentaron complicaciones como falla orgánica multisistémica $(20,4$ $\%)(n=2)$, choque séptico $(30,4 \%)(n=3)$, paro cardiorrespiratorio $(15,9 \%)$ $(n=3)$, falla respiratoria $(22,7 \%)(n=2)$ o falla renal aguda $(29,5 \%)(n=3)$; en 


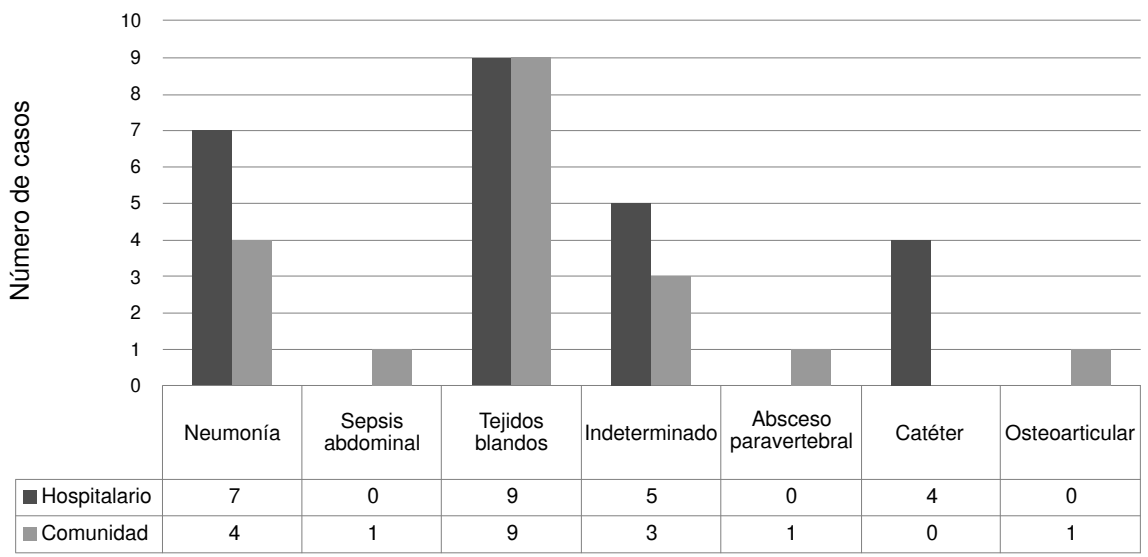

Figura 1. Distribución de los focos de infección primaria asociados con la bacteriemia según sitio de adquisición

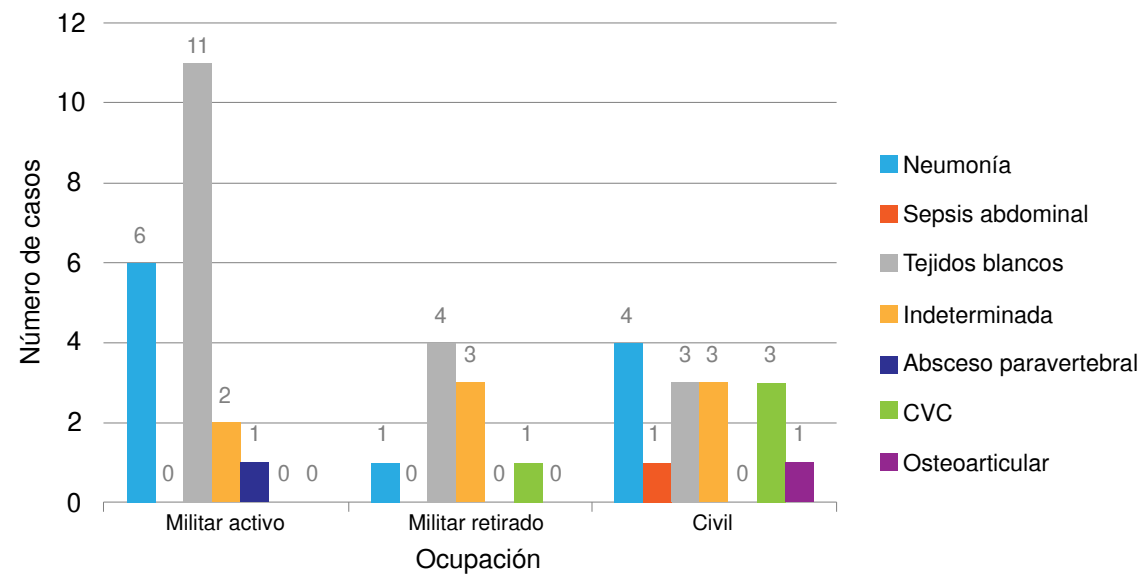

Figura 2. Distribución de focos de infección primaria asociados con la bacteriemia según la profesión

$29,5 \%(n=3)$ de los casos, la bacteriemia los condujo a la muerte. En cuatro individuos $(9,1 \%)$, se diagnosticó fazscitis necrosante y embolia séptica.

La mortalidad fue mayor en el grupo con bacteriemia por SAMR de origen hospitalario que en el de la adquirida en la comunidad (40\% Vs. 15,8\%) [( $n=10)$ $V s$. $(n=3)]$. En el análisis por subgrupos de fenotipo, se encontró una mortalidad de $42,8 \%(n=3)$ por HA-MRSA, en tanto que, por CA-MRSA, fue de $27 \%(n=10)$.

Por último, en cuanto a la estancia hospitalaria, se encontró que esta fue prolongada en $34,9 \%(n=15)$ de los casos y que podía atribuirse a la bacteriemia por SAMR, con un promedio de 36,9 $\pm 14,6$ días; los porcentajes fueron similares entre los pacientes con bacteriemia adquirida en la comunidad y aquellos que la adquirieron en el ámbito hospitalario, 31,5\% $(n=6)$ y $36 \%(n=9)$, respectivamente.

\section{Discusión}

En este estudio, se caracterizó un grupo de pacientes con diagnóstico de bacteriemia por hemocultivos positivos para SAMR. En la revisión de Aiello, et al. (13) de estudios en dos grupos de alto riesgo, presos y 
militares, en que se evaluaban la prevalencia y los factores de riesgo de la infección por SAMR, los autores incluyeron el estar en la cárcel, el sexo, las comorbilidades, la infección previa de la piel y el uso previo de antibióticos.

Aunque características como la higiene, el contacto físico y el hacinamiento se han postulado como factores de riesgo para la infección por SAMR, hubo pocos estudios epidemiológicos que respaldaran dicho planteamiento. La mayoría de los estudios encontrados tenía un diseño retrospectivo y solo en uno se recurrió a la vigilancia prospectiva para la colonización de SAMR en todos los individuos que residían en un solo entorno militar. En otros países, se han reportado casos en la población militar (14-23). No obstante, en nuestro país no hay información estadística en este sentido hasta la fecha.

Del total de hemocultivos positivos $(n=177)$ para $S$. aureus, el $24,8 \%$ $(n=44)$ correspondía a SAMR, hallazgo que discrepa con lo reportado en otras latitudes con prevalencias de hasta de 59,2\%, por ejemplo, en los Estados Unidos (5). En el presente estudio, se encontró que la frecuencia de bacteriemia por SAMR adquirida en la comunidad era similar a la de la adquirida en el hospital, con $43,1(n=19)$ y $56,8 \%(n=25)$, respectivamente; en tanto que, en otros reportes, se registra un aumento en la incidencia progresiva de las infecciones por SAMR adquiridas en la comunidad (24-28).

Una de las limitaciones de este estudio consistió en que no se disponía de confirmación genotípica para el subtipo SCCmec de SAMR, responsable de la resistente a la meticilina. Sin embargo, se hizo una caracterización fenotípica basada en el perfil de resistencia en el antibiograma, y se observó una alta prevalencia $(84,1 \%, n=37)$ de cepas con fenotipo CA-MRSA, tanto en la bacteriemia adquirida en la comunidad como en la hospitalaria. El fenotipo HA-MRSA se relacionó con una mayor mortalidad, semejante a lo documentado en otros estudios.

En diversos estudios, se ha comparado la eficacia de las técnicas de laboratorio convencionales y de las moleculares en la identificación de SAMR. Los resultados han demostrado una equivalencia de 90 a $95 \%$ entre las diferentes series reportadas, lo que inclina la balanza a favor de la detección mediante pruebas convencionales que, en términos de costoefectividad, superan a las herramientas moleculares. Sin embargo, estas son necesarias para identificar clones en los estudios epidemiológicos (29-31).

En su estudio, Wang, et al., determinaron los factores de riesgo asociados con la mortalidad en las bacteriemias por SAMR y, mediante genotipificación del subtipo $\mathrm{SSCmec}$, encontraron una adecuada correlación entre el patrón fenotípico de resistencia y el genotipo de SAMR (1). En este estudio, se observó una mortalidad similar a los 14 y a los 30 días con el subtipo HA-MRSA.

En el presente estudio, los tejidos blandos fueron el foco de infección más frecuentemente relacionado con el desarrollo de bacteriemia por SAMR, seguido del pulmonar (figura 1). En el estudio de Laupland, et al., los principales focos infecciosos se localizaron en las zonas pélvica y en la abdominal (25\%), el aparato respiratorio (16\%), y los tejidos blandos (10\%), así como los debidos a intervenciones endovasculares (8\%) o los huesos y articulaciones (7\%) (15). Aunque en ambos estudios la mayoría de los focos de infección primaria fueron los mismos, se registraron diferencias en cuanto a la frecuencia de presentación, lo cual podría tener relación 
con el tipo de población evaluada, ya que, en el presente estudio, la mayor frecuencia se registró en pacientes militares activos, personal que está expuesto a las lesiones de piel.

La bacteriemia asociada con el uso de catéter venoso central en la población aquí estudiada, se presentó en 9,09 \% $(n=4)$ de los pacientes y representó el $16 \%(n=4)$ de las bacteriemias por SAMR de origen hospitalario, en tanto que, en la literatura médica, se han reportado casos hasta en el $39 \%$ (16).

En 56,82\% ( $n=25)$ de la población estudiada, se encontró algún tipo de comorbilidad en el momento del ingreso al hospital, como diabetes mellitus $(20,45 \%)(n=5)$, inmunosupresión farmacológica $(15,6 \%)(n=4)$ por quimioterapia o por uso crónico de corticoides, e insuficiencia renal (9\%) $(n=2)$. El $44 \%$ de estos pacientes tuvo complicaciones relacionadas con la bacteriemia durante su estancia hospitalaria; las más frecuentes fueron la falla orgánica multisistémica y la insuficiencia renal aguda, además de los casos de muerte. Estos hallazgos se asemejan a los reportados en la literatura médica, siendo las comorbilidades de mayor impacto la enfermedad renal, los tumores sólidos, la diabetes mellitus con uso de insulina y el HIV $(5,14)$.

Según las guías para el manejo y seguimiento de los pacientes con bacteriemia por SAMR (14), se deben hacer hemocultivos a las 48 y las 72 horas de inicio del tratamiento antibiótico, así como ecocardiografía para determinar la gravedad de la bacteriemia, establecer la duración del tratamiento antibiótico y determinar la presencia de endocarditis infecciosa $(3,5,14,17)$. Es necesario optimizar el cumplimiento de estas guías de manejo.

Todos los pacientes evaluados recibieron tratamiento antibiótico empírico y, en $79,5 \%(n=35)$ de la población con actividad contra SAMR, se administró tratamiento con un solo antibiótico o combinado con otros, siendo la vancomicina el más utilizado. Una vez se obtuvo la tipificación del microorganismo y se confirmó la presencia de SAMR en los hemocultivos, el antibiótico más utilizado continuó siendo la vancomicina, en $50 \%(n=22)$ de los casos. Sin embargo, llamó la atención el uso del tratamiento combinado $(40,90 \%)(n=16)$, siendo la combinación de un betalactámico y la vancomicina $(15,91 \%)(n=7)$ la más frecuente, lo que podría generar mayores costos y complicaciones derivadas del uso inadecuado de estos medicamentos.

Estos hallazgos justifican la implementación de un programa de uso prudente de los antibióticos, que hoy hace parte de las estrategias institucionales de control de las infecciones.

Los medicamentos de primera línea aprobados por la US Food and Drug Administration (FDA) para la bacteriemia por SAMR, son la vancomicina y la daptomicina, y la utilización de una u otra se decide según la concentración mínima inhibitoria de la vancomicina. En varios estudios se ha sugerido la utilización de la daptomicina ante la presencia de altas concentraciones mínimas inhibitorias de la vancomicina. El trimetropim-sulfametoxazol y el linezolid pueden ser útiles cuando han fallado los antibióticos de primera línea.

Asimismo, se recomienda que todos los pacientes sean tratados por vía intravenosa (17). Recientemente, se ha querido comparar la eficacia entre el manejo entérico y el parenteral (32). El estudio SABATO, actualmente en curso, aún no arroja reportes definitivos; su principal objetivo es demostrar que, en pacientes con bacteriemia por $S$. aureus de bajo riesgo, el cambio del tratamiento antimicrobiano intravenoso al oral no reduce la eficacia contra la infección (33). 
Los sesgos posibles del presente estudio incluyen el no haber hecho la identificación genotípica y la evaluación de la mortalidad atribuible al aislamiento comparada con el peso de las comorbilidades y su gravedad.

Aproximadamente, el 24,8\% $(n=44)$ de los aislamientos de $S$. aureus en hemocultivos correspondían a SAMR en la población de estudio, y se registró una frecuencia similar entre las bacteriemias adquiridas en la comunidad y las adquiridas en el ámbito hospitalario.

En este estudio, se encontró que los militares activos fueron los más afectados por este microrganismo, y el principal foco infeccioso fueron los tejidos blandos. Se necesitan estudios posteriores que permitan determinar la prevalencia de SAMR como causa de infección de los tejidos blandos para, así, evaluar la necesidad de establecer protocolos que incluyan fármacos de primera línea en el tratamiento antibiótico empírico (vancomicina o daptomicina) de las bacteriemias por SAMR en este contexto.

La frecuencia de la bacteriemia por SAMR asociada con el uso de catéter central fue baja $(9 \%, n=4)$ en nuestra población. Se presentó un $61 \%(n=13)$ de complicaciones en la bacteriemia de origen hospitalario, siendo la más frecuente la falla orgánica multisistémica.

El fenotipo CA-MRSA representó la causa más frecuente $(80,4 \%, n=37)$ de bacteriemia en el Hospital Militar, con prevalencia del fenotipo HAMRSA. Sin embargo, se requieren estudios que verifiquen los genotipos para corroborar este dato.

El seguimiento de los pacientes con diagnóstico de bacteriemia por SAMR fue deficiente y, aunque no se contempló como una variable del estudio, se pudo observar que es necesario implementar estrategias de uso prudente de los antibióticos, así como evaluar su cumplimiento. El tratamiento antibiótico combinado utilizado de forma empírica debe evaluarse en el momento de la confirmación microbiológica de SAMR, ya que puede generar mayores costos innecesarios.

\section{Referencias}

1. Wang JT, Wang JL, Fang CT, Chie WC, Lai MS, Lauderdale TL, et al. Risk factors for mortality of nosocomial methicillin-resistant Staphylococcus aureus (MRSA) bloodstream infection: With investigation of the potential role of community-associated MRSA strains. J Infect. 2010;61:449-57. https://doi.org/10.1016/j.jinf.2010.09.029

2. Grupo para el Control de la Resistencia Bacteriana de Bogotá (GREBO). Resultados de la vigilancia de la resistencia bacteriana, año 2016. Componente pediátrico y adulto. Análisis de percentiles marcadores de resistencia 2016. Tendencias de los principales marcadores de resistencia, 2008-2016. Boletín GREBO. 2017;9:1-23.

3. Jiménez JN, Ocampo AM, Vanegas JM, Rodríguez EA, Mediavilla JR, Chen L, et al. A comparison of methicillin-resistant and methicillin-susceptible Staphylococcus aureus reveals no clinical and epidemiological but molecular differences. Int J Med Microbiol. 2013;303:76-83. https://doi.org/10.1016/j.ijmm.2012.12.003

4. Gasch O, Camoez M, Domínguez MA, Padilla B, Pintado V, Almirante B, et al. Predictive factors for mortality in patients with methicillin-resistant Staphylococcus aureus bloodstream infection: Impact on outcome of host, microorganism and therapy. Clin Microbiol Infect. 2013;19:1049-57. https://doi.org/10.1111/1469-0691.12108

5. Naber CK. Staphylococcus aureus bacteremia: Epidemiology, pathophysiology, and management strategies. Clin Infect Dis. 2009;48(Suppl.4):S231-7. https://doi.org/10.1086/598189

6. Chong YP, Park S, Kim HS, Kim ES, Kim M, Park K, et al. Persistent Staphylococcus aureus bacteremia, a prospective analysis of risk factors, outcomes and microbiologic and genotypic characteristics of isolates. Medicine (Baltimore). 2013;92:98-108. 
7. Lam SW, Bauer SR, Neuner EA. Predictors of septic shock in patients with methicillinresistant Staphylococcus aureus bacteremia. Int J Infect Dis. 2012;16:e453-6. https://doi. org/10.1016/j.ijid.2012.02.007

8. Taylor AR. Methicillin-resistant Staphylococcus aureus infections. Prim Care. 2008;16:63754. https://doi.org/ 10.1016/j.pop.2013.06.002

9. Pastagia M, Kleinman LC, Lacerda EG, Cruz D, Jenkins SG. Predicting risk for death from MRSA bacteriemia. Emerg Infect Dis. 2012;18:1072-80. https://doi.org/10.3201/eid1807.101371

10. Corey GR. Staphylococcus aureus bloodstream infections: Definitions and treatment. Clin Infect Dis. 2009;27715(Suppl.4):S254-9. https://doi.org/10.1086/598186.11

11. Naves SK, Vaz N, Pinto P, Filho G. Methicillin-resistant Staphylococcus aureus bloodstream infection: Risk factors and clinical outcome in non-intensive-care units. Rev Soc Bras Med Trop. 2012;45:189-93. https://doi.org/10.1590/S0037-86822012000200010

12. Popovich K, Hota B, Rice T, Aroutcheva A, Weinstein RA. Phenotypic prediction rule for community-associated methicillin-resistant Staphylococcus aureus. J Clin Microbiol. 2007;45:2293-5. https://doi.org/10.1128/JCM.00044-07

13. Aiello A, Lowy E, Wright FD, Larson L. Methicillin-resistant Staphylococcus aureus among US prisoners and military personnel: Review and recommendations for future studies. Lancet Infect Dis. 2006;6:335-41. https://doi.org/10.1016/S1473-3099(06)70491-1

14. Zinderman C, Conner B, Malakooti M, LaMar J, Armstrong A, Bohnker B. Communityacquired methicillin-resistant Staphylococcus aureus among military recruits. Emerg Infect Dis. 2004;10:941-4. https://doi.org/10.3201/eid1005.030604

15. Washington MA, Agee WA 3rd, Kajiura L, Staege CM, Uyehara CF, Barnhill JC. An analysis of Staphylococcus aureus infections at a military medical center using the PLEX-ID combined polymerase chain reaction-mass spectrometry system. Mil Med. 2014;179:44550. https://doi.org/10.7205/MILMED-D-13-00472

16. Vento T, Calvano T, Cole D, Mende K, Rini E, Tully CH, et al. Staphylococcus aureus colonization of healthy military service members in the United States and Afghanistan. BMC Infect Dis. 2013;13:325. https://doi.org/10.1186/1471-2334-13-325

17. Hospenthal D, Green A, Crouch H, English J, Pool J, Yun H, et al. Infection prevention and control in deployed military medical treatment facilities. J Trauma. 2011;71:S290-8. https://doi.org/10.1097/TA.0b013e318227add8

18. Braun L, Craft D, Williams R, Tuamokumo F, Ottolini M. Increasing clindamycin resistance among methicillin-resistant Staphylococcus aureus in 57 Northeast United States Military treatment facilities. Pediatr Infect Dis J. 2005;24:622-6. https://doi.org/10.1097/TA.0b013e318227add8

19. Landrum M, Neumann $\mathrm{CH}$, Cook C, Chukwuma U, Ellis M, Hospenthal D, et al. Epidemiology of Staphylococcus aureus blood and skin and soft tissue infections in the US Military Health System, 2005-2010. JAMA. 2012;308:50-9. https://doi.org/10.1001/jama.2012.7139

20. Roberts S, Kazragis R. Methicillin-resistant Staphylococcus aureus infections in U.S. service members deployed to Iraq. Mil Med. 2009;174:408.

21. Sherwood J, Park M, Robben P, Whitman T, Ellis M. USA 300 Methicillin-resistant Staphylococcus aureus. Emerging as a cause of bloodstream infections at military medical centers. Infect Control Hosp Epidemiol. 2013;34:393-9. https://doi.org/10.1086/669866

22. Whitman TJ, Herlihy RK, Schlett CD, Murray P. Chlorhexidine-impregnated cloths to prevent skin and soft-tissue infection in marine recruits: A cluster-randomized, double-blind, controlled effectiveness trial. Infect Control Hosp Epidemiol. 2010;31:1207-15. https://doi.org/10.1086/657136

23. Ganesan A, Crawford K, Mende K, Murray C, Lloyd B, Ellis M, et al. Evaluation for a novel methicillin resistance $(\mathrm{mecC})$ homologue in methicillin-resistant Staphylococcus aureus isolates obtained from injured military personnel. J Clin Microbiol. 2013;51:3073-5. https://doi.org/10.1128/JCM.01516-13

24. Paul M, Kariv G, Goldberg E, Raskin M, Shaked H, Hazzan R, et al. Importance of appropriate empirical antibiotic therapy for methicillin-resistant Staphylococcus aureus bacteraemia. J Antimicrob Chemother. 2010:2658-65. https://doi.org/10.1093/jac/dkq373

25. Laupland KB, Ross T, Gregson DB. Staphylococcus aureus bloodstream infections: Risk factors, outcomes, and the influence of methicillin resistance in Calgary, Canada, 20002006. J Infect Dis. 2008;198:336-43. https://doi.org/10.1086/589717 
26. Arias-Ortiz PM, del Pilar-Calderón L, Castillo JS. Factores de riesgo de la resistencia a meticilina de Staphylococcus aureus causante de bacteriemia: Un estudio multicéntrico de casos y controles emparejados. Biomédica. 2016;36:612-8. https://doi.org/10.7705/biomedica.v36i4.3193

27. Holland TL, Arnold C, Fowler VG. Clinical management of Staphylococcus aureus bacteremia. JAMA. 2014;312:1330. https://doi.org/10.1001/jama.2014.9743

28. International Working Group on the Classification of Staphylococcal Cassette Chromosome Elements (IWG-SCC). Classification of Staphylococcal cassette chromosome mec (SCCmec): Guidelines for reporting novel SCCmec elements. Antimicrob Agents Chemother. 2009;53:4961-7. https://doi.org/10.1128/AAC.00579-09

29. Wang WY, Chiueh TS, Sun JR, Tsao SM, Lu JJ. Molecular typing and phenotype characterization of methicillin-resistant Staphylococcus aureus isolates from blood in Taiwan. PLoS One. 2012;7:e30394. https://doi.org/10.1371/journal.pone.0030394

30. Gradelski E, Valera L, Aleksunes A, Bonner D, Fung-Tomc J. Correlation between genotype and phenotypic categorization of Staphylococci based on methicillin susceptibility and resistance. J Clin Microbiol. 2001;39: 2961-3. https://doi.org/10.1128/JCM.39.8.2961-2963.2001

31. Clinical and Laboratory Standards Institute. CLSI Guidelines. Performance Standards for Antimicrobial Susceptibility Testing; Sixteenth Informational Supplement to CLSI Approved Standard M2-A8, Performance Standards for Antimicrobial Disk Susceptibility Tests. Wayne, PA: CLSI; 2016.

32. López-Cortés LE, de Cueto M, Rodríguez-Baño J. How should we best treat patients with bloodstream infections? Future Microbiol. 2017;12:927-30. https://doi.org/10.2217/fmb-2017-0118

33. Kaasch A, Fätkenheuer G, Prinz-Langenohl R, Paulus U, Hellmich M, Wei V, et al. Early oral switch therapy in low-risk Staphylococcus aureus bloodstream infection (SABATO): Study protocol for a randomized controlled trial. Trials. 2015;16:450.

https://doi.org/10.1186/s13063-015-0973-x 